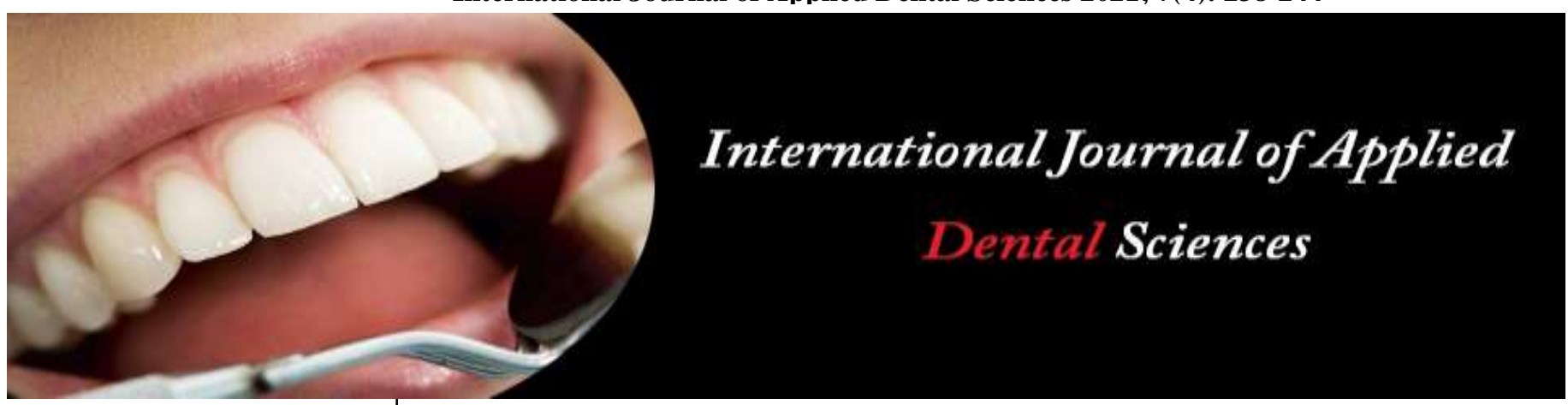

ISSN Print: 2394-7489

ISSN Online: 2394-7497

IJADS 2021; 7(4): 238-244

(C) 2021 IJADS

www.oraljournal.com

Received: 13-08-2021

Accepted: 15-09-2021

Dr. Faizul Ariff Sahul Hamid General Dentist and British Commonwealth Scholar, Bin Arab Dental and Medical Centre, Flat no 102, Al Othaiba building, Al Rigga Road

Deira, Dubai, United Arab Emirates
Corresponding Author: Dr. Faizul Ariff Sahul Hamid General Dentist and British Commonwealth Scholar, Bin Arab Dental and Medical Centre, Flat no 102, Al Othaiba building, Al Rigga Road Deira, Dubai, United Arab Emirates

\section{Dental management considerations in the elderly with acquired bleeding disorders}

\section{Dr. Faizul Ariff Sahul Hamid}

DOI: https://doi.org/10.22271/oral.2021.v7.i4d.1377

\section{Abstract}

Background: Globally, the percentage of elder people is increasing every year and has been projected that by year 2050 one in six people in the world will be 65 years and older. With advancing age, there are increased changes in the hemostatic balance resulting in acquired bleeding disorders. It has become very common for such patients to seek oral healthcare in hospitals and clinics. Hence it is essential that Dentists are able to manage such patients with regards to ensuring patient safety and delivering high standards of care.

Objective: Enable Dental professionals to recognize underlying hematological comorbidities, order and interpret appropriate lab investigations, identify drugs which might cause bleeding during dental treatment, planning and delivering required dental procedures especially invasive procedures such as dental extractions.

Methods: Literature review searches were done PubMed, Wiley Online \& Cochrane Library Databases using keywords.

Keywords: Acquired bleeding disorders, elderly population, aging, dental treatment considerations

\section{Introduction}

The advancements in medical healthcare systems and practices have led to a global longevity revolution. Along with this increased life expectancy there is also an increase in the comorbidities in older population with bleeding disorders being one of them. These bleeding disorders can be congenital or acquired. Congenital bleeding disorders are genetic in nature and present at birth while acquired bleeding disorders develop after birth or emerge at any point in life ${ }^{[1]}$. Usually, geriatric patients will be aware of their underlying congenital bleeding disorders and will readily discuss them with their treating dentist. However, acquired bleeding disorders in older people poses serious challenges to the treating dentist. These disorders are not readily diagnosed, their early signs overlooked as consequences of aging or at times the patients themselves might not be aware of their existence as they tend to develop spontaneously ${ }^{[2]}$.

Due to current population trends, older people can be expected to constitute a significant proportion of any dental practice. Hence Dentists need to recognize the balance between the provision of dental care and the oral bleeding such procedures might cause. This article is an attempt at discussing some of the challenges in provision of dental care to such patients and means to overcome them.

\section{Global trends in Aging}

Human population aging is a global success phenomenon reflecting advances in medicine, public health, social and economic development which have greatly reduced the risk of premature death ${ }^{[3]}$. 
According to the United Nations Report on World population aging 2019, the number of people worldwide aged 65 and above is projected to grow by $120 \%$ between 2019 and 2050 . This growth percentage is expected to be around $197.9 \%$ for people who are 80 years and above in the same time frame. These projections indicate that the number of people aged 80 and above shall grow at a faster rate than people aged 65 and above in the next three decades worldwide. Therefore, Dentists should be able to treat the elderly bearing in mind the multiple medical conditions, psychological, social and familial factors that might influence provision of care.

Table 1: United Nations Global geriatric population. Growth projections: 2019 to 205Q

\begin{tabular}{|c|c|c|c|c|c|c|}
\hline \multirow[t]{2}{*}{ Regiem } & \multicolumn{2}{|c|}{$\begin{array}{l}\text { Number of persons aged } 65 \\
\text { years or over in millions }\end{array}$} & \multirow{2}{*}{\begin{tabular}{|c|} 
Percentage \\
change between \\
2019 and 2050 in \%
\end{tabular}} & \multicolumn{2}{|c|}{\begin{tabular}{|c|} 
Number of persons aged 80 \\
years or over in millions \\
\end{tabular}} & \multirow{2}{*}{$\begin{array}{c}\text { Percentage change } \\
\text { between } 2019 \text { and } \\
2050 \text { in } \%\end{array}$} \\
\hline & 2019 & 2050 & & 2019 & 2050 & \\
\hline World & 702.9 & 1548.9 & \begin{tabular}{|l|}
120 \\
\end{tabular} & 143.1 & 426.4 & 197_0 \\
\hline Sub-Saharan Africa & 31.9 & 101.4 & 218 & 3.7 & 12.4 & 238.1 \\
\hline Northern Africa and Western Asia & 29.4 & 95.8 & 226 & 5.2 & 20.3 & 291 \\
\hline Central and Southern Asia & 119.0 & 328.1 & 176 & 18.5 & 62.6 & 239.0 \\
\hline Eastern and South-Eastern Asia & 260.6 & 572.5 & 120 & 48.6 & 177.0 & 264.1 \\
\hline Latin America and the Caribbean & 56.4 & 144.6 & 156 & 12.0 & 41.4 & 245.2 \\
\hline Australia and New Zealand & 4.8 & 8.8 & 84 & 1.2 & 3.3 & 168.4 \\
\hline $\begin{array}{l}\text { Oceania excluding Australia and New } \\
\text { Zealand }\end{array}$ & 0.5 & 1.5 & 190 & 0.1 & 0.2 & 269.1 \\
\hline Europe and Northern America & 200.4 & 296.2 & 48 & 53.9 & 109.1 & 102.6 \\
\hline
\end{tabular}

Source: United Nations Department of Economic and Social Affairs, Population Division 2019 world population prospects 2019

\section{Etiology and prevalence of acquired bleeding disorders in the elderly}

Bleeding or coagulation disorders like Hemophilia and von Willebrand disease are caused by abnormalities in coagulation factors and may be congenital or acquired. On the other hand, platelet disorders like idiopathic thrombocytopenic purpura and thrombocytopenia are caused by abnormalities in platelet form and function.

Haemostasias changes with aging due to a number of factors. Most coagulation factors and platelet activation increase with age which results in increased thrombin generation ${ }^{[4]}$. Aging is also associated with decreased fibrinolytic activity, [5] changes in vascular endothelium and impaired renal function. All these factors influence coagulation and hemostatic agent interactions in the elderly. The common acquired bleeding disorders in the elderly are drug induced, secondary to renal impairment/chronic kidney disease, acquired Hemophilia and acquired von Willebrand's disease.

\section{Drug induced}

Bleeding in older people is commonly iatrogenic. There is greater use of NSAIDs and aspirin to treat chronic pain associated with aging. Risk of venous thromboembolism and cardiovascular disease in elderly necessitates use of antiplatelet drugs (Aspirin, Clopidogrel) and anticoagulant drugs (Heparin, Warfarin) which increases bleeding risk ${ }^{[6]}$ in older people.

\section{Renal disease}

Older people are at greater risk of developing chronic kidney disease (CKD) due to risk factors like diabetes, hypertension and cardiovascular disease ${ }^{[7]}$. As of 2018, researchers at John Hopkins University USA have estimated that globally 1 in 5 men and 1 in 4 women between ages 65 and 74 years and more than $50 \%$ of people aged 75 or more have chronic kidney disease ${ }^{[8]}$. There is an increased bleeding tendency in CKD patients, probably due to a functional platelet disorder
[9]. The resultant uremic platelets along with associated anemia results in increased perioperative bleeding in CKD patients ${ }^{[10]}$.

\section{Acquired Hemophilia A (AH)}

Acquired hemophilia $\mathrm{A}$ is characterized by spontaneous bleeding in patients with no prior history of any bleeding disorders ${ }^{[11]}$. It is caused by the development of IgG auto antibodies to endogenous Factor VIII mostly due to an underlying malignancy or autoimmune disorder. Among older population, $50 \%$ of $\mathrm{AH}$ patients are idiopathic with an average age of 73.9 years at diagnosis ${ }^{[12]}$. Acquired Hemophilia A in older people poses a challenge to the treating dentist as it tends to be undiagnosed probably due to coexistence with other bleeding conditions ${ }^{[13]}$.

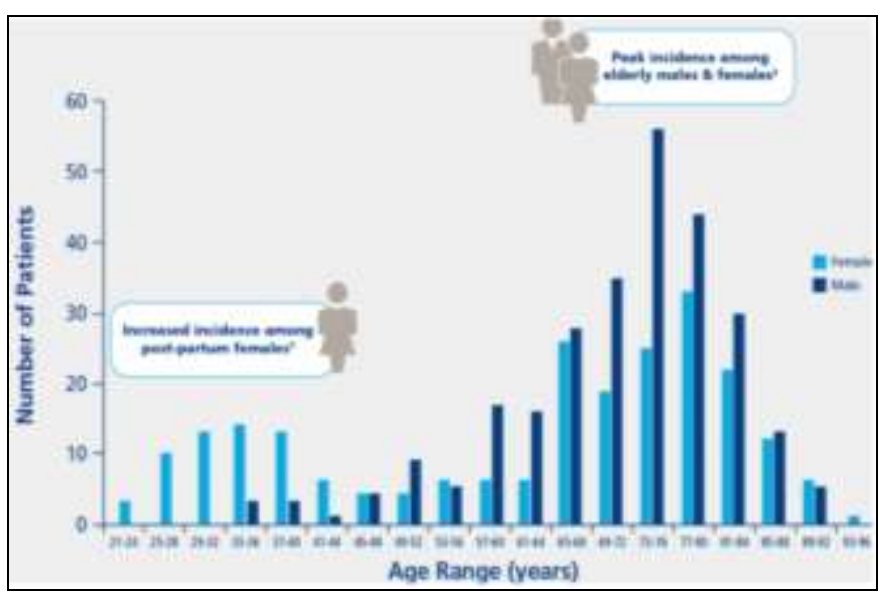

Source: Demographic and clinical data in acquired hemophilia A: results from the European Acquired Hemophilia Registry (EACH2). J Thromb Haemost. 2012;10(4):622-631.

Fig 1: Prevalence of Acquired Hemophilia A by age and gender in Europe 


\section{Acquired von Willebrand syndrome (AVWS)}

Acquired von Willebrand syndrome is a rare disorder caused by increased clearance of von Willebrand factor either by antibodies, increased lysis or decreased synthesis. AVWS has been shown to be exclusively linked to an underlying disorder ${ }^{[14]}$ like cardiovascular or myeloproliferative disorders ${ }^{[15]}$. Even though this disorder has been seen in children, there are increasing evidence of its presentation with advancing age. The average age at presentation was 64,68 and 62 years for people with lymphoproliferative, cardiovascular disorders or neoplasms as underlying cause ${ }^{[16]}$. However, it has been seen that AVWS resolves with treatment of the underlying cause.

\section{Dental treatment considerations History and Evaluation}

A negative bleeding history to previous surgical procedures or trauma can eliminate congenital bleeding disorders but might not safely rule out acquired bleeding disorders in the elderly. The American Dental association recommends the following additional questions to be asked to a patient with suspicion of any bleeding disorder.

\section{Table 2 - ADA medical listory questiomaire for suspected bleeding disorden}

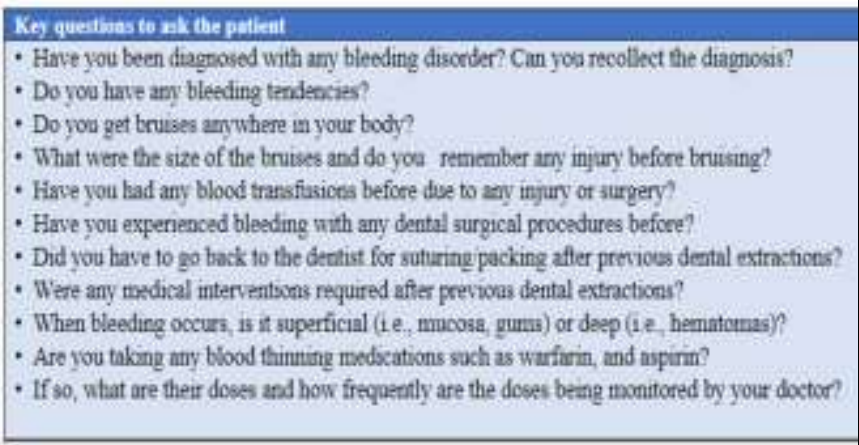

Source: Patton, Lauren L. The ADA practical guide to patients with medical conditions. John Wiley \& Sons 2015:183-200.

Affirmative responses to disorders necessitating blood thinning medications or any of the above questions should alert the dentist to initiate further enquiries with the patient's physician prior to designing and implementing any dental treatment plans.

\section{Dentist Physician Coordination}

Provision of dental care to older patients with a diagnosed or suspected bleeding disorder requires collaboration between the dentist and patient's physician prior to performing any dental procedure. Apart from the primary physician, the team can include other professionals like a cardiologist, or hematologist depending upon the nature and severity of the underlying disorder. Dental treatment plans might require alterations and/or measures prior to, during and following dental procedures specifically formulated based on medical advice.
The American Dental association recommends the following questions to be asked to a physician providing medical care to a patient with a diagnosed or suspected bleeding disorder.

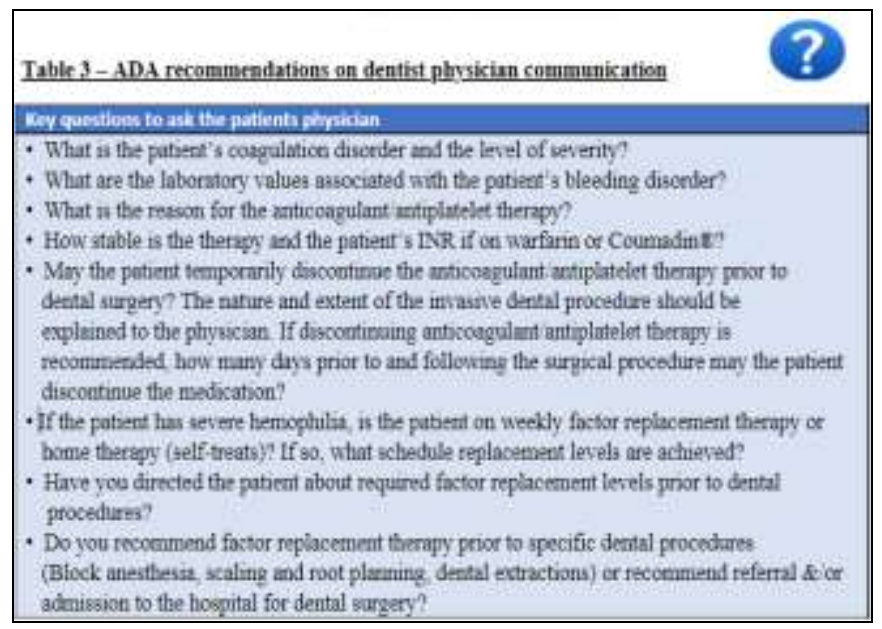

Source: Patton, Lauren L. The ADA practical guide to patients with medical conditions. John Wiley \& Sons, 2015:183-200 ${ }^{[17]}$

With key questions answered, communication established between the dentist and the physician, investigations can be performed and dental treatment plans be formulated bearing in mind the severity of the bleeding disorder.

\section{Laboratory investigations}

The ability to order and interpret laboratory bleeding tests is essential if a dentist is to provide surgical dental care to older people with any bleeding disorder. The common blood tests advocated to assess bleeding disorders are summarized in the following table.

Table 4: Laboratory investigations used to assess hemostasis

\begin{tabular}{|c|c|c|}
\hline DAlsoratury Tet & $\overline{\text { Nurmal Ratrye }}$ & What if nextiures \\
\hline \multicolumn{3}{|l|}{ Compien hlocd canei } \\
\hline Wented Count & $\begin{array}{l}150,000-40.000 \\
\text { celle mit }\end{array}$ & Fatelet qualiny \\
\hline Fy biedine time? & Less than 6 mia & Patrilet functian \\
\hline FADT00 & Closure time 993 , & 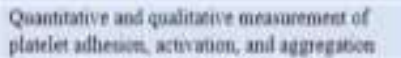 \\
\hline $\bar{r}$ & $11-145$, & $\begin{array}{l}\text { Facten } 11 \text { (prothembin), V, VU, and } \mathrm{X} \text {, and } \\
\text { Gluinesen }\end{array}$ \\
\hline EQR & 1.0 & \\
\hline arts & $27-31 \mathrm{~s}$ & Factoes II, V, VIII, IX, X XI, and XII \\
\hline 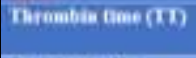 & $9-13 \mathrm{~s}$ & 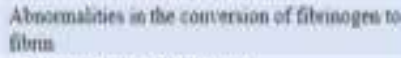 \\
\hline Antipartar $\mathrm{Xa}$ & 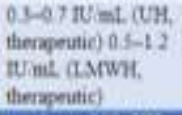 & Fasma $\mathrm{CH}$ and LMWH levels \\
\hline \multicolumn{3}{|c|}{ 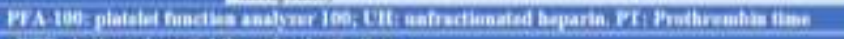 } \\
\hline Arris Metivated pant & 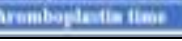 & \\
\hline
\end{tabular}

Source: Journal of Family and Community medicine. 2007;14(2):53-58 ${ }^{[18]}$.

In addition to the basic tests, there are advanced tests used in the diagnosis of $\mathrm{AH}$ and AVWS which are summarized in the following table. 


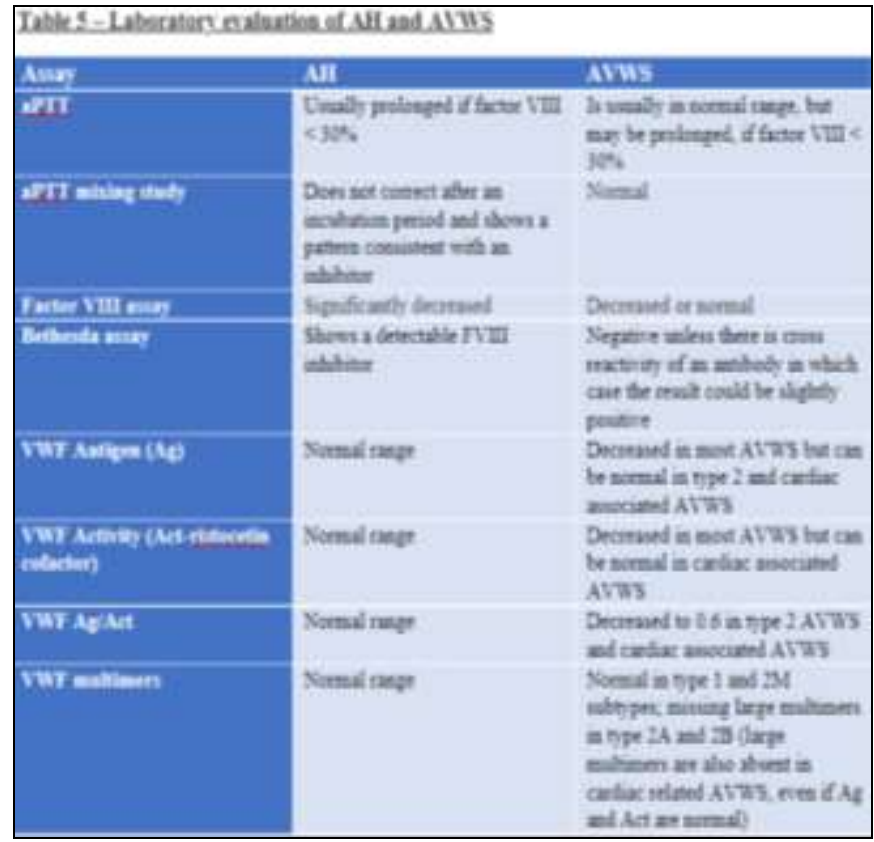

Source: American Society of Hematology Education Program Book 2015;1(2015):231-236.

\section{Socio-economic considerations and informed consent}

Chronologic age cannot be considered as a definitive criterion in geriatric dentistry due to significant heterogenicity in ageing population with respect to physical \& mental health, socio-economic status and disparities in oral health. In 1894, Ettinger and suggested the division of ageing population into following three groups based on their ability to seek dental services ${ }^{[19]}$.

1. Functionally independent older adults - live unassisted in the community, have sone chronic medical problems and can access dental services by using own or public transportation.

2. Frail older adults - includes people who have lost some independence but live-in communities with family or friends. These people can no longer access dental services without the help of others.

3. Functionally dependent older adults- includes people cannot live independently in the community and are either home bound or live-in care homes. They require transportation and accompanying people to seek dental services and might require wheelchair assistance or even home visits by the dentist to provide care.

In addition, the provision of dental care also depends on other factors like perceived treatment need - patient, spouse \& immediate relatives, people providing activities of daily living support and the financial considerations for the proposed treatment plan.
It is crucial that the dentist is able to communicate with elderly patients and their families. It has been shown that older patients tend to ask fewer questions to their treating physicians even though they seek more clarifications ${ }^{[20]}$. This is due to sensory and hearing difficulties along with probable cognitive decline which greatly influence informed consent capabilities ${ }^{[21]}$. Hence the dentist must make every effort to convey the oral health status, proposed treatment plan, costs and any other special requirements to the elderly patients and their families.

\section{Preventive dentistry concepts}

Reports worldwide have highlighted the low use of professional dental services by older people ${ }^{[22]}$. The presence of medical comorbidities and socio-economic drawbacks further complicates the provision of dental care. The following preventive concepts can be recommended by dentists to older patients to try and avoid any possible future ailments.

- Regular periodic professional oral health evaluation

- Topical fluoride mouthwashes and applications - reduce root caries lesions in older people ${ }^{[23]}$.

- Topical fluoride applications with chlorhexidine rinsing reduces tooth loss in elderly ${ }^{[24]}$

- Chlorhexidine rinses reduce gingival inflammation, pocket depth \& denture stomatitis ${ }^{[25]}$.

- Use of electric toothbrushes, adaptive grip modifications of toothbrushes \& home care aids

- Professional oral health education improves oral health in older people ${ }^{[26]}$.

- Group based behavior modification intervention improved brushing and flossing skills while reducing gingival bleeding in older people ${ }^{[27]}$.

\section{Local anesthesia and pain management}

The use of local anesthetics in older people at the dental office are considered safe when administered properly. There are no significant differences in responses to their administration in the elderly ${ }^{[28]}$. Use of Local anesthetic agents with vasoconstrictors are also advocated as they provide additional hemostasis. However, it is advised to limit the dose of epinephrine in anesthetics to a maximum of 0.04 mg considering the effects of aging on the heart ${ }^{[29]}$.

It has been shown that hemophilia patients have an $80 \%$ chance of developing hematoma following an inferior alveolar nerve block without prior factor VIII cover ${ }^{[30]}$. This hematoma can be catastrophic as it compromises the airway and accumulates in the mediastinum ${ }^{[31]}$. Improper needle placement during a posterior superior alveolar nerve blocks also have a tendency to cause hematoma due to trauma of the pterygoid venous plexus ${ }^{[32]}$.

The dentist should discuss prophylactic preoperative factor 
VIII cover with the patient's hematologist prior to administering inferior dental \& posterior superior nerve blocks, lingual or floor of the mouth infiltration in any bleeding disorder patient ${ }^{[33]}$. However, mental nerve block buccal infiltration, intrapulpal and intraligamentary anesthetic techniques are safer and do not require factor VIII cover ${ }^{[34]}$.

Paracetamol can be safely used to control dental pain. However, since non-steroidal anti-inflammatory drugs (NSAIDs) effect platelet aggregation, prior consultation with patient's hematologist is necessary before use.

\section{Age related oral mucosal changes and dental considerations}

Aging induced oral mucosal changes results in decreased sensitivity to various stimuli, loss of taste and reduced healing potential following mucosal injury. The reactions of oral mucosa to any mechanical trauma like ill-fitting prosthesis change with aging. Such trauma invokes a painful inflammatory response in younger individuals while chronic atrophic processes predominate in the elderly ${ }^{[35]}$. The following measures can prevent iatrogenic injury to the tissues while carrying out a dental procedure as they have the potential to cause complications in older people with bleeding disorders.

- Careful use of saliva ejectors, covering suction tips with gauze ${ }^{[36]}$

- Applying soft paraffin (vaseline ${ }^{\circledR}$ ) before using rubber dam, matrix bands and wedge ${ }^{[37]}$.

- Using Nonmetallic impression trays, exercising care when removing impressions from mouth

- Consider measures like air abrasion, intra oral scanning $\&$ chemo mechanical caries removal ${ }^{[38]}$.

- Use of stable finger rests, good intra oral retractors \& intra oral camera

- Ensure proper engagement of needle to syringe body prior to use.

- Avoid excessive pressure during intracanal irrigation

\section{Dental procedure guidelines}

Drug induced bleeding disorders

Older patients with drug induced (Aspirin, NSAIDs) functional platelet bleeding disorders do not require alterations on dosage for minor dental procedures [39]. However, for patients on oral anticoagulants (Warfarin) dental treatments can be planned with the patient's hematologist considering the patients International Normalized Ratio (INR) and the nature of the proposed procedure. The following table illustrates the guidelines for performing outpatient dental procedures on warfarin therapy.

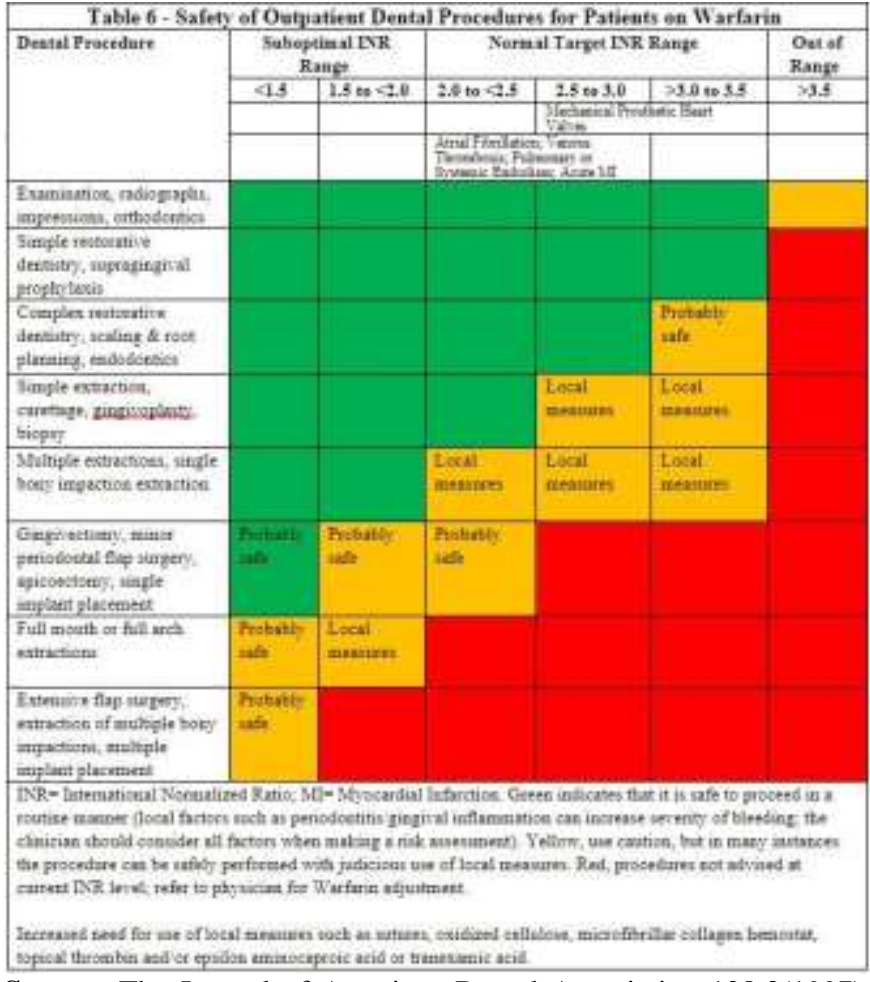

Source: The Journal of American Dental Association 128.3(1997): $327-355^{[40]}$.

The hematologist might recommend withholding warfarin for at least 24 hours or longer prior to the dental procedure ${ }^{[41]}$. For more extensive dental procedures requiring multiple visits, the hematologist might suggest substituting warfarin with low molecular weight heparin ${ }^{[42]}$.

\section{Drug induced, AH and AVWS}

For elderly patients with AH and AVWS, the following prophylaxis regimens can be advocated by a hematologist prior to any dental surgical procedure.

- Platelet \& fresh frozen plasma transfusions, Vitamin K (iv/sc) administration

- Protamine sulfate (Heparin antagonist) administration ${ }^{[43]}$

- Desmopressin acetate (iv/sc/intranasal), recombinant factor VIII administration ${ }^{[44]}$ one hour

- $\quad$ prior to dental procedure

- Maintain factor VIII levels - 50-75\% before minor oral and periodontal surgery, 75-100\%

- before maxillofacial surgery ${ }^{[45]}$

- Oral rinse for 2 minutes with $5 \%$ Tranexamic acid mouthwash

- Antibiotic prophylaxis 
The following protocols can be implemented by a dentist during and after dental treatment based on the procedure.

\section{Dental extraction \& periodontal surgery}

- Flapless atraumatic tooth extraction whenever possible

- Use Resorbable hemostatic dressing - oxidized cellulose (Sugicel), Gelatin (Gelfoam)

- Resorbable sutures with simple suture techniques, bite pressure with gauze pack

- Use of preformed vacuum splint to protect socket if necessary

- Provide written \& verbal instructions to patients and their caregivers.

- Emergency dental/hospital contact numbers to be provided - for post-operative emergencies

- Advice $5 \%$ Tranexamic acid mouthwash 4 times /1-gram tablets 3 times - daily for 7-10 days

Other Dental procedures like supragingival scaling, full \& partial prosthodontics \& endodontic treatments are generally considered safe for elderly patients with bleeding disorders. It is advisable to design restorations and fixed prosthesis with supra gingival margins and trim sharp edges in clasp wires and other restorations that might injure oral tissues and cause bleeding.

\section{Conclusion}

Oral health is an integral part of general health and wellbeing of elderly. Dentists must understand the influence of social and medical factors on oral health which in turn influences the design and provision of dental treatment. The occurrence of $\mathrm{AH}$ and AVWS in older people are rare and are often not diagnosed which can lead to fatal consequences. Hence dentists must employ a team approach to care provision which involves summative risk assessments and designing a treatment plan which ensures optimal oral health which fits into the comprehensive needs of older people.

\section{References}

1. Doherty TM, Kelley A. Bleeding Disorders. [Updated 2021 Aug 12]. In: StatPearls [Internet]. Treasure Island (FL): StatPearls Publishing 2021. Available from: https://www.ncbi.nlm.nih.gov/books/NBK541050/

2. Kruse-Jarres, Rebecca. "Acquired bleeding disorders in the elderly." Hematology 2014, the American Society of Hematology Education Program Book 2015;1:231-236.

3. United Nations, Department of Economic and Social Affairs, Population Division 2020. World Population Ageing 2019 (ST/ESA/SER.A/444).

4. Favaloro, Emmanuel J, Massimo Franchini, Giuseppe Lippi. "Aging hemostasis: changes to laboratory markers of hemostasis as we age-a narrative review." Seminars in thrombosis and hemostasis. Thieme Medical Publishers 2014;40:06.

5. Ocak Gürbey, et al. "Role of hemostatic factors on the risk of venous thrombosis in people with impaired kidney function." Circulation 2014;129(6):683-691.

6. Lamberts Morten et al. "Relation of nonsteroidal antiinflammatory drugs to serious bleeding and thromboembolism risk in patients with atrial fibrillation receiving antithrombotic therapy: A nationwide cohort study." Annals of internal medicine 2014;161(10):690698.

7. Williams Mark E. "Diabetic kidney disease in elderly individuals." Medical Clinics 2013;97(1):75-89.
8. Source;http;//thelancet.com/pb/assets/raw/pbassets/raw/la ncet/campaigns/kidney/chronic-kidney-disease-facts.jpg 99-18-10856-01-76 (C) Siemens Healthcare Diagnostics Inc;2018

9. Mannucci, Pier Mannuccio, Armando Tripodi. "Hemostatic defects in liver and renal dysfunction." Hematology 2010, the American Society of Hematology Education Program Book 2012;(1):168-173.

10. Acedillo Rey R et al. "The risk of perioperative bleeding in patients with chronic kidney disease: A systematic review and meta-analysis." Annals of surgery 2013;258(6):901-913.

11. Goldman Lee, Andrew Schafer I. Goldman's cecil medicine E-book. Elsevier Health Sciences, 2011.

12. Knoebl $\mathrm{P}$ et al. "Demographic and clinical data in acquired hemophilia A: results from the European Acquired Hemophilia Registry (EACH2)." Journal of Thrombosis and Hemostasis 2012;10(4):622-631.

13. Source: Acquired hemophilia. [Aug;2019];https://rarediseases.org/rarediseases/acquired-hemophilia/ 2019

14. Federici Augusto B et al. "Current diagnostic and therapeutic approaches to patients with acquired von Willebrand syndrome: A 2013 update." Seminars in thrombosis and hemostasis. Thieme Medical Publishers 2013;39:02.

15. Tiede Andreas. "Diagnosis and treatment of acquired von Willebrand syndrome." Thrombosis research 2012;130:S2-S6.

16. Federici Augusto B et al. "Acquired von Willebrand syndrome: data from an international registry." Thrombosis and hemostasis 2000;84(08):345-349.

17. Patton Lauren L. The ADA practical guide to patients with medical conditions. John Wiley \& Sons 2015, 183200

18. Bashawri Layla AM, Mirghani Ahmed A. "The approach to a patient with a bleeding disorder: for the primary care physician." Journal of family \& community medicine 2007;14(2):53.

19. Ettinger Ronald L, James Beck D. "Geriatric dental curriculum and the needs of the elderly." Special care in dentistry 1984;4(5):207-213.

20. Rudd Rima, Alice Horowitz M. "The role of health literacy in achieving oral health for elders." Journal of dental education 2005;69(9):1018-1021.

21. Yellowitz Janet A. "Cognitive function, aging, and ethical decisions: recognizing change." Dental Clinics 2005;49(2):389-410.

22. Petersen Poul Erik, Tatsuo Yamamoto. "Improving the oral health of older people: the approach of the WHO Global Oral Health Program." Community dentistry and oral epidemiology 2005;33(2):81-92.

23. Wallace Martha C, Hugo Retiet D, Edwin Bradley L. "The 48-month increment of root caries in an urban population of older adults participating in a preventive dental program." Journal of public health dentistry 1993;53(3):133-137.

24. Wyatt CCL, MacEntee MI. Caries management for institutionalized elders using fluoride and chlorhexidine mouth rinses. Community Dent Oral Epidemiol 2004;32:322-8.

25. Persson Rigmor E, et al. "Therapeutic effects of daily or weekly chlorhexidine rinsing on oral health of a geriatric population." Oral surgery, oral medicine, oral pathology 1991;72(2):184-191. 
26. Haas Daniel A. "An update on local anesthetics in dentistry." Journal-Canadian Dental Association 2002;68(9):546-552.

27. Schou Lone. "Oral health, oral health care, and oral health promotion among older adults: social and behavioral dimensions." Disease prevention and oral health promotion. Copenhagen: Munksgaard 1995, 21370.

28. Little SJ, et al. "Effective group behavioral intervention for older periodontal patients." Journal of periodontal research 1997;32(3):315-325.

29. Haas Daniel A. "An update on local anesthetics in dentistry." Journal-Canadian Dental Association 2002;68(9):546-552.

30. Ouanounou Aviv, Daniel Haas A. "Pharmacotherapy for the elderly dental patient." J Can Dent Assoc 2015;80:18.

31. Webster WP, Roberts HR, Penick GD. "Dental care of patients with hereditary disorders of blood coagulation." Modern treatment 1968;5(1):93-110.

32. Heiland Max, Martin Weber, Rainer Schmelzle. "Lifethreatening bleeding after dental extraction in a hemophilia A patient with inhibitors to factor VIII: a case report1." Journal of oral and maxillofacial surgery 2003;61(11):1350-1353.

33. Malamed Stanley F. Handbook of local anesthesia. Elsevier Health Sciences, 2004.

34. Srivastava Alok, et al. "Guidelines for the management of hemophilia." Haemophilia 2013;19(1):e1-e47.

35. Rafique Sobia, et al. "Special care dentistry: part 1. dental management of patients with inherited bleeding disorders." Dental update 2013;40(8):613-628.

36. Breustedt A. "Age-induced changes in the oral mucosa and their therapeutic consequences." International dental journal 1983;33(3):272-280.

37. Robertson Douglas, et al. "The anesthetic efficacy of articaine in buccal infiltration of mandibular posterior teeth." The Journal of the American Dental Association 2007;138(8):1104-1112.

38. Katz Jerald O, Geza Terezhalmy T. "Dental management of the patient with hemophilia." Oral surgery, oral medicine, oral pathology 1988;66(1):139-144.

39. Leal SC. Minimum intervention in dentistry: applicability for dental care of patients with inherited bleeding disorders [abstract 11S02]. Haemophilia 2010;16(4):50.

40. Napeñas Joel J, et al. "Review of postoperative bleeding risk in dental patients on antiplatelet therapy." Oral surgery, oral medicine, oral pathology and oral radiology 2013;115(4):491-499.

41. Herman Wayne W, Joseph Konzelman Jr L, Stephen Sutley H. "Current perspectives on dental patients receiving coumarin anticoagulant therapy." The Journal of the American Dental Association 1997;128(3):327335.

42. Firriolo John F, Wendy Hupp S. "Beyond warfarin: the new generation of oral anticoagulants and their implications for the management of dental patients." Oral surgery, oral medicine, oral pathology and oral radiology 2012;113(4):431-441.

43. Douketis James D, Judith Johnson A, Alexander Turpie G. "Low-molecular-weight heparin as bridging anticoagulation during interruption of warfarin: assessment of a standardized periprocedural anticoagulation regimen." Archives of Internal Medicine 2004;164(12):1319-1326.

44. Love, Donald G. "Management of hemorrhagic events in patients receiving anticoagulant therapy." Journal of thrombosis and thrombolysis 1999;7(2):149.

45. Anderson JAM, et al. "Guidance on the dental management of patients with haemophilia and congenital bleeding disorders." British dental journal 2013;215(10):497-504.

46. Scully Crispian, Pedro Diz Dios, Navdeep Kumar. Special care in dentistry e-book: Handbook of Oral Healthcare. Elsevier Health Sciences, 2006. 